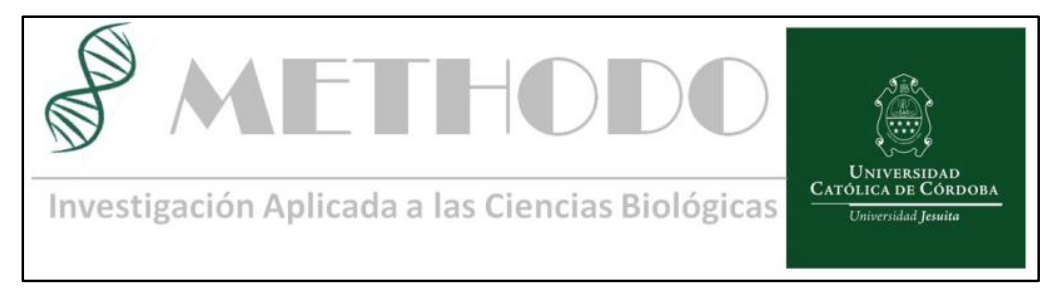

CASO CLINICO Methodo 2017;2(4): 129-133

DOI: 10.22529/me.2017.2(4)08

\title{
Rehabilitación protésica implanto-soportada en un paciente con epidermolisis ampollar distrófica recesiva. 4 años de seguimiento clínico
}

\section{Implant-supported dental prostheses in a patient with recessive dystrophic epidermolisis bullosa. Four-year follow- up.}

\author{
Molina $\mathrm{G}^{1}$, Torassa $\mathrm{D}^{1}$, Ribba $\mathrm{L}^{1}$.
}

\begin{abstract}
Resumen Se describe la evolución de una paciente con diagnóstico molecular de Epidermolisis Ampollar Distrófica Recesiva, quien a los 35 años de edad inicia una rehabilitación bucal mediante prótesis fija implanto-soportada, luego de haber realizado las exodoncias de las piezas remanentes en boca. Todos los implantes se mantuvieron oseointegrados a lo largo de cuatro años de seguimiento clínico y no se encontró flora periodontopática peri-implantar en los controles microbiológicos realizados. Este tipo de rehabilitación es un caso paradigmático para personas con este tipo de genodermatosis, constituyéndose en una posibilidad válida para una mejor calidad de vida frente a las circunstancias adversas que plantea la enfermedad.

Palabras claves: Genodermatosis, Implantes Dentales, Rehabilitación Oral, Epidermolisis Ampollar
\end{abstract}

\begin{abstract}
Oral rehabilitation of a 35-years old female patient with Recessive Dystrophic Epidermolisis Bullosa is described. After removal of all remaining teeth, cemented implantasisted prosthesis with shortened arches were installed over three implants in the mandible and four implants in the upper jaw. At the four- year follow up, all the implants conserved osseointegration as well as absence of peri-implantar periodontopatic biofilm was observed. The success achieved in this case becomes a paradigmatic landmark for the oral rehabilitation of patients with such genodermatoses, providing a new resource to improve their quality of life.
\end{abstract}

Keywords: Genodermatoses, Dental Implants, Oral Rehabilitation, Epidermolisis Bullosa

1. Carrera de Odontología, Facultad de Ciencias de la Salud, Universidad Católica de Córdoba Correspondencia: Gustavo Molina. Libertad 1282 B ${ }^{\circ}$ General Paz - 5000 Córdoba, Argentina. Te. 0351-4462330 e-mail: gfmolina@ hotmail.com 
Molina G, Torassa D, Ribba L. Rehabilitación protésica implanto-soportada en un paciente con epidermolisis ampollar distrófica recesiva. 4 años de seguimiento clínico

\section{Introducción}

La atención odontológica en personas con discapacidad es una disciplina en constante crecimiento, la cual requiere del aporte interdisciplinario que permita un abordaje integral del ser humano en sus diferentes dominios. Si bien este marco filosófico podría aplicarse a todas las disciplinas de la salud, es especialmente relevante en el diagnóstico, la prevención y la planificación terapéutica de personas con patologías en las que las interrelaciones disciplinares son eslabones esenciales para apuntalar la calidad de vida.

La Epidermolisis Bullosa o Ampollar (EB) responde a un desorden genético autosómico caracterizado por la aparición de lesiones ampollares inducidas por el roce, afectando especialmente zonas de la piel y mucosa sujetas a fricción. Estas ampollas se ulceran y posteriormente cicatrizan de manera anormal. La EB se agrupa dentro de las llamadas "genodermatosis" y puede presentarse en tres tipos: simple, de la unión y distrófica, siendo esta última forma en su modo recesivo la que manifiesta un mayor compromiso de la mucosa oral $^{1}$.

La Epidermolisis Ampollar Distrófica Recesiva (RDEB) representa uno de los mayores desafíos para la prevención y tratamiento de las enfermedades de la boca. Las patologías orales más frecuentes en la RDEB consisten en repetidas ampollas y erosiones en la mucosa que conducen gradualmente a la reducción de la apertura bucal, de los movimientos linguales y del surco vestibular. La pérdida precoz de piezas dentarias por causa de caries o de enfermedad periodontal estimula la resorción ósea y la atrofia de los maxilares ${ }^{2}$.

La paciente que se describe en este reporte presenta una situación representativa de la evolución de la salud bucal en personas con RDEB, ya que es posible constatar el deterioro sufrido en su boca a lo largo de su vida. En función de las necesidades extremas de la paciente, se analizó la posibilidad y luego se llevó a cabo una rehabilitación protésica mediante la fijación a implantes dentales de férulas métalo-cerámicas de arco reducido en ambas arcadas dentarias. Si bien existe evidencia de rehabilitaciones protésicas implanto soportadas en pacientes con RDEB realizadas en Europa $^{3-5}$, este es el primer reporte de una experiencia llevada a cabo en Sudamérica.

\section{Reporte del caso}

Una paciente de 35 años de edad, género femenino, con diagnóstico molecular de Epidermolisis Ampollar Distrófica Recesiva, se presentó a la consulta odontológica motivada por el temor a perder sus incisivos inferiores, los cuales evidenciaban signos de movilidad compatibles con una periodontitis severa. (Fig. 1)

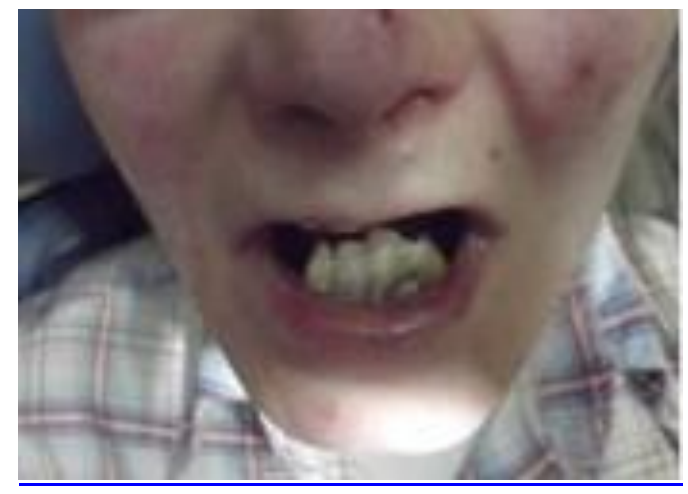

Figura 1. Observación clínica extraoral del estado de salud bucal de la paciente.

La inspección clínica de la cavidad bucal permitió observar una reducción de la apertura bucal (microstomia), con $22 \mathrm{~mm}$ de distancia entre los bordes incisales de ambas arcadas y $38 \mathrm{~mm}$ entre las comisuras labiales. La movilidad lingual se presentaba limitada significativamente y la mucosa oral se despegaba fácilmente ante un moderado roce produciendo nuevas ampollas y ulceraciones. Se constató la movilidad de los dientes del sector anterior, afectados por caries macropenetrantes y por enfermedad periodontal severa. La información provista por la imagen de la radiografía panorámica de su boca reveló la existencia de restos radiculares en la región posterior de los maxilares (Fig. 2).

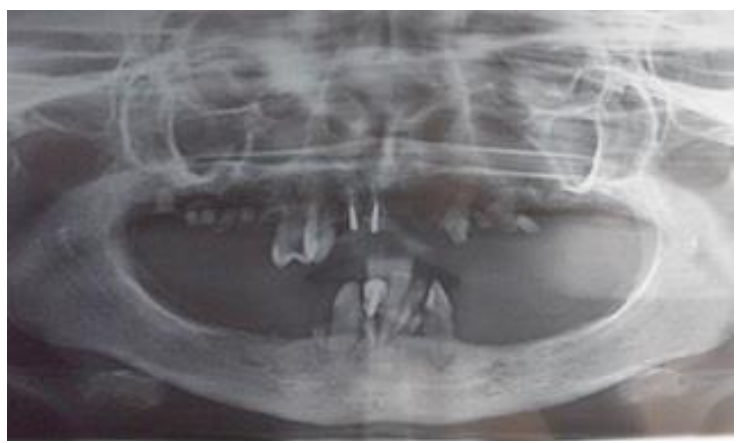

Figura 2. Ortopantomografía inicial en la cual se observa el estado de salud bucal y la presencia de restos radiculares en el sector postero-superior.

En una primera instancia, la posibilidad de anclaje protésico en remanentes dentarios no se contempló como una alternativa factible, considerando que la pérdida de sus piezas dentarias estaba relacionada a caries o a enfermedad periodontal severa. En tal sentido, se planteó como necesaria la realización de 
Molina G, Torassa D, Ribba L. Rehabilitación protésica implanto-soportada en un paciente con epidermolisis ampollar distrófica recesiva. 4 años de seguimiento clínico

extracciones de los remanentes dentarios para planificar una etapa de rehabilitación protésica, pero la opción de una prótesis removible mucosoportada no se consideró adecuada para una persona con tal fragilidad en sus mucosas orales. El hallazgo de evidencia bibliográfica sobre el uso exitoso de implantes dentales para la rehabilitación bucal de personas con RDEB fue un elemento de valoración a la hora de una propuesta de prótesis fija, la cual quedó supeditada a la posibilidad de colocar implantes en los maxilares de la paciente una vez finalizada la etapa de remoción de todas las piezas remanentes y la regularización de los rebordes alveolares.

Luego de dos meses de haber realizado las exodoncias se solicitó un estudio de Tomografía Computada tipo Cone Bean (CBCT) a partir del cual se definió un abordaje quirúrgico sin colgajo para la instalación de mini-implantes categoría I según la clasificación de Klein y col. ${ }^{6}$ (monoimplantes de $<3.0 \mathrm{~mm}$ ). De este modo, sin necesidad de una intervención bajo anestesia general u otro tipo de sedación, utilizando los mismos anestésicos locales para infiltración profunda que se habían empleado para las exodoncias (Carticaína con epinefrina, Totalcaína, Laboratorios Bernabó, Argentina), con fecha 20 de Mayo de 2013 se colocaron 3 implantes en la arcada inferior y 4 implantes en la superior, de $2.4 \mathrm{~mm}$ de diámetro y $10 \mathrm{~mm}$ de longitud con esferas para anclaje (implantes transitorios IT, ByW, Argentina), con un período de vigilancia de 14 semanas previa a la carga de la prótesis. Durante este lapso, la temporización fue realizada con prótesis removibles sostenidas en forma precaria por las esferas de anclaje de los mono-implantes.

Una vez superada la etapa de oseointegración, se procedió a la carga de la prótesis a través de férulas métalo-cerámicas de arco reducido, reponiendo 8 elementos por arcada. Para asegurar la adaptación de las piezas coladas se realizaron controles preliminares de ajuste en materiales calcinables, permitiendo la adecuación de las estructuras y la estimación de la dimensión vertical para el montaje en articulador semi-adaptable. De este modo, las dificultades en la toma de registro fueron sorteadas hasta alcanzar la mayor precisión posible en los pasos de construcción protésica.

Los puentes definitivos fueron cementados provisoriamente con un cemento para tal fin (Protemp, 3M Espe, Seefeld, Alemania) con la intención de brindar a la paciente la oportunidad de evaluar la rehabilitación en funcionamiento, de manera que los posibles ajustes pudieran llevarse a cabo con anterioridad a la fijación definitiva. Durante este período de prueba se seleccionaron junto a la paciente los recursos más adecuados para el mantenimiento de la salud periodontal, controlando la formación de un biofilm con potencial patogénico en la zona periimplantar. En tal sentido, se optó por los Softpicks (GUM, Buttler Co, Alemania) para remoción mecánica de placa y enjuagues con Clorhexidina al $0.12 \%$ (Plac-Out Colutorio, Laboratorios Bernabó, Argentina) para control químico, realizando los procedimientos de higiene bucal luego de cada comida.

Finalmente, los puentes se cementaron con un cemento de ionómero vítreo tipo I (G-Cem, GC America, Chicago, EE UU), controlando su fijación cada 6 meses tanto en una evaluación clínica como radiográfica. (Fig. 3) La oseointegración se mantuvo exitosa para los 7 implantes a lo largo de 4 años de seguimiento.

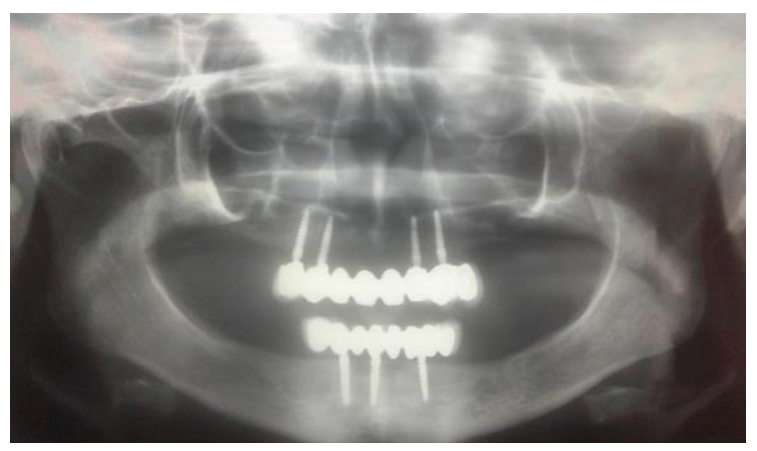

Figura 3. Ortopantomografía de control en la que se observan los 7 implantes oseointegrados y las férulas cementadas sobre los pilares.

En las sucesivas citas de control se observó desgaste de la porción cerámica a la altura del elemento 12 y sus antagonistas 42 y 43, revelando un área de desgaste por movimientos parafuncionales. En consecuencia, se realizaron los desgastes correspondientes, evaluando nuevamente los contactos durante las trayectorias de lateralidad mandibular. Después de dichos ajustes, se pulieron las superficies desgastadas con discos y gomas para tal fin. (Fig. 4)

Se tomaron muestras de biofilm peri-implantar con conos de papel número 35 durante 15 a 20 segundos, colocando rápidamente el material en tubos Eppendorf con un contenido de $2 \mathrm{ml} \mathrm{de}$ medio de transporte estéril RTF conservado a $4^{\circ} \mathrm{C}$. Se procedió a la siembra y cultivo de las muestras en agar Columbia preparado según las indicaciones del fabricante, a baño de agua a 45$50^{\circ} \mathrm{C}$ durante 30 minutos, luego esterilizado en autoclave, dejándose enfriar finalmente en baño de agua hasta retomar $\operatorname{los} 45-50^{\circ} \mathrm{C}$. Se incorporó sangre de cordero al $5 \%$ final $(\mathrm{v} / \mathrm{v})$, y $3 \mathrm{ml}$ de hemina-menadiona al medio estéril bajo campana de flujo laminar hasta alcanzar los 
Molina G, Torassa D, Ribba L. Rehabilitación protésica implanto-soportada en un paciente con epidermolisis ampollar distrófica recesiva. 4 años de seguimiento clínico
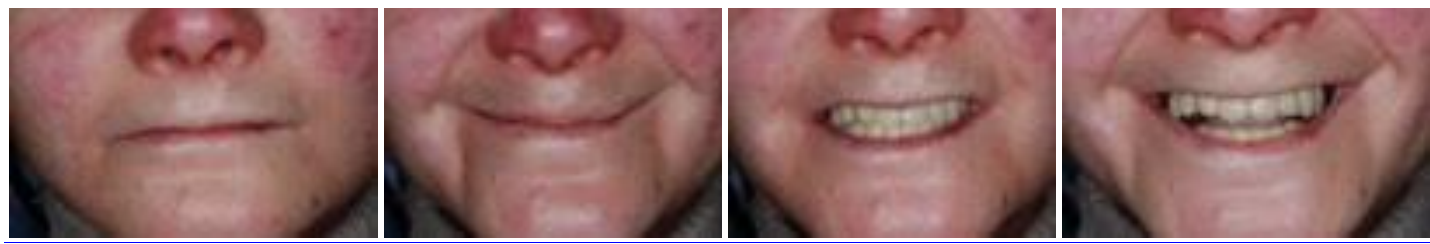

Figura 4. Control clínico en el que se observan los desgastes en el sector del 12/42-43.

$300 \mathrm{ml}$ de medio total; luego se dispensaron 20ml de medio por placa para realizar cultivos e identificar el desarrollo de cepas bacterianas periodontopáticas asociadas a posibles respuestas inflamatorias alrededor de los implantes. Estas muestras fueron procesadas en el Laboratorio de Microbiología de Clínica Universitaria Reina Fabiola y los correspondientes controles microbiológicos anuales no revelaron el desarrollo de un biofilm con presencia de flora patogénica para la instalación de peri-implantitis.

\section{Discusión}

En la Odontología actual se ha consolidado la tendencia a tomar decisiones clínicas basadas en una combinación proporcional de la experiencia del operador y la evidencia científica que existe sobre el problema a resolver. Esto es válido para todas las disciplinas aplicadas a ciencias de la salud, pero ciertamente contradictorio en situaciones raras o de baja prevalencia. Como lo señala Bergendal (2006), es en estas situaciones donde los reportes de casos y avances terapéuticos, cuya relevancia ocupa uno de los niveles más bajos en la escala de evidencia científica, cobran significativa importancia a la hora de una construcción colaborativa y colectiva del conocimiento universal ${ }^{7}$.

En tal sentido, la colocación de implantes como alternativa para la fijación de una rehabilitación mediante prótesis fija en una paciente con RDEB resultaba un desafío con pronóstico reservado. La existencia de publicaciones científicas con buenos resultados fue determinante para que los autores decidieran avanzar sobre este abordaje ${ }^{3-}$ 5 , aprovechando las experiencias previas sobre los problemas que debieron sortear otros colegas en situaciones similares.

No obstante, cada caso plantea dificultades particulares tales como el grado de apertura bucal para la instalación de los implantes, la altura y el ancho de los rebordes óseos donde serán colocados, el diseño protésico en función de los implantes seleccionados y su localización en las arcadas, además de las posibilidades para la toma de impresiones y registros con cierta fidelidad en la reproducción del terreno quirúrgico y protésico. Las decisiones que se tomaron para cada etapa de la planificación terapéutica fueron la respuesta a estas particularidades.

En cuanto a las impresiones y registros, se sugirió la posibilidad que la reproducción del terreno quirúrgico y protésico se lograra a través de recursos digitales con la utilización de Tomografías Computadas y la impresión en 3D de los tejidos duros y blandos. Agustín-Panadero y col. reportan la incorporación de esta tecnología como un aporte significativo para la reproducción de los tejidos con exactitud, sin forzar ni lesionar pliegues mucosos ${ }^{8}$. El acceso a este tipo de recursos permitiría superar la limitación en apertura bucal, evitando la posibilidad de provocar ampollas por roces de las mucosas o el efecto de succión generado por los materiales de impresión. Lamentablemente, la imposibilidad para acceder a esta tecnología obligó a la resolución del caso mediante el manejo artesanal de los materiales tradicionales que se utilizan para la toma de impresiones y registros, es decir, cubetas individualizas a las arcadas de la paciente, elastómeros de consistencia pesada y fluida, reproducciones en yeso de alta resistencia y montaje en articulador semi-adaptable luego de esbozar los registros de mordida con la prueba de las estructuras calcinables.

En una revisión de publicaciones referidas a la colocación de implantes en pacientes con trastornos en mucosa, se enumeran 16 pacientes en el grupo de Epidermolisis Ampollar entre 13 y 52 años de edad, en los que un total de 92 implantes fueron controlados durante 1 a 9 años, con porcentajes de éxito cercanos al 100\% (sólo 1 estudio de 6 reportó $75 \%$ de sobrevida). En todos los casos el tipo de rehabilitación protésica se inclinó a la instalación de sobredentaduras o férulas fijas de arco reducido ${ }^{9}$.

En el caso reportado en este artículo, se optó por mono-implantes como la mejor alternativa para lograr oseointegración en espesores de hueso muy reducidos. Su distribución en los rebordes alveolares estuvo orientada por las imágenes que se obtuvieron tanto en la ortopantomografía como en la tomografía Cone-bean, en las cuales el cálculo del ancho de los rebordes no alcanzaba para alojar implantes de mayor diámetro. 
Molina G, Torassa D, Ribba L. Rehabilitación protésica implanto-soportada en un paciente con epidermolisis ampollar distrófica recesiva. 4 años de seguimiento clínico

En un mono-implante se integra en una sola pieza, por un lado, el anclaje al hueso a través del implante y, por el otro, el pilar o muñón para la fijación de la prótesis. En función de haber planteado una rehabilitación fija cementada, se utilizaron los pilares (cuyo diseño original era para prótesis removible implanto-mucosoportada con sistema ball-attach) para que actuaran como muñones para la fijación de una férula cementada sobre éstos.

De igual manera, los mono-implantes utilizados condicionaron la ejecución de una prótesis fija cementada en lugar de una atornillada ${ }^{10}$. Esta última posibilita realizar ajustes ante las eventualidades que surgen tanto de la función masticatoria como de los hábitos parafuncionales, tal como ocurrió en el caso descripto por Muller y col. ${ }^{3}$, quienes debieron retirar las estructuras protésicas fracturadas por bruxismo para replantear la rehabilitación frente a los cambios producidos en el terreno.

\section{Bibliografía}

1. Babu NA, Rajesh E, Krupaa J, Gnananandar G. Genodermatoses. J Pharm Bioallied Sci. 2015 Apr;7(Suppl 1):S203-6. doi: 10.4103/09757406.155903. Review.

2. Dağ C, Bezgin T, Özalp N. Dental management of patients with epidermolysis bullosa. Oral Health Dent Manag. 2014 Sep;13(3):623-7.

3. Muller F, Bergendal B, Wahlmann U, Wagner W. Implant-supported fixed dental prostheses in an edentulous patient with dystrophic epidermolysis bullosa. Int J Prosthodont. 2010 Jan-Feb;23(1):42-8.

4. Peñarrocha-Oltra D, Peñarrocha-Diago $M$, BalaguerMartínez J, Ata-Ali J, Peñarrocha-Diago M. Full-arch fixed prosthesis supported by four implants in patients with recessive dystrophic epidermolysis bullosa. Oral Surg Oral Med Oral Pathol Oral Radiol Endod. 2011 Aug;112(2):e4-10. doi: 10.1016/j.tripleo.2011.03.022.

5. Agustín-Panadero R, Gomar-Vercher S, PeñarrochaOltra D, Guzmán-Letelier M, Peñarrocha-Diago M. Fixed full-arch implant-supported prostheses in a patient with epidermolysis bullosa: a clinical case history report. Int J Prosthodont. 2015 JanFeb;28(1):33-6. doi: 10.11607/ijp.4092.
Independientemente del tipo de implante $\mathrm{y}$ diseño protésico llevado a cabo en los diferentes reportes publicados, en todos los casos se resaltan altos niveles de satisfacción por parte de los pacientes, lo cual se traduce una percepción concreta de mejor calidad de vida por parte de los beneficiarios de estos tratamientos. En este punto también hubo coincidencia con el impacto generado en la paciente que se describe en el presente artículo.

\section{Conclusión}

El resultado de una rehabilitación mediante prótesis fija implanto-soportada evaluado a lo largo de 4 años de seguimiento de una paciente con diagnóstico de Epidermolisis Ampollar Distrófica Recesiva, sumado a la evidencia de otros reportes en la bibliografía internacional, posiciona a esta alternativa terapéutica como un recurso que contribuye a mejorar significativamente la calidad de vida de estas personas.
6. Maryod WH, Ali SM, Shawky AF. Immediate versus early loading of mini-implants supporting mandibular overdentures: a preliminary 3-year clinical outcome report. Int J Prosthodont. 2014 Nov-Dec;27(6):55360. doi: 10.11607/ijp.3845.

7. Bergendal B Evidence and clinical improvement: current experiences with dental implants in individuals with rare disorders. Int J Prosthodont 2006 Mar-Apr; 19(2):132-4.

8. Agustín-Panadero R, Serra-Pastor B, PeñarrochaOltra D, Peñarrocha-Diago M. Maxillary Implant Prosthodontic Treatment Using Digital Laboratory Protocol for a Patient with Epidermolysis Bullosa: A Case History Report. Int J Prosthodont. 2017 Jul/Aug;30(4):390-393. doi: 10.11607/ijp.5065.

9. Candel-Marti ME, Ata-Ali J, Peñarrocha-Oltra D, Peñarrocha-Diago M, Bagán JV Dental implants in patients with oral mucosal alterations: An update. Med Oral Patol Oral Cir Bucal. 2011 Sep 1;16(6):e787-93.

10. Wittneben JG, Millen C, Brägger U. Clinical performance of screw- versus cement-retained fixed implant-supported reconstructions--a systematic review. Int J Oral Maxillofac Implants. 2014;29 Suppl:84-98. doi: 10.11607/jomi.2014suppl.g2.1. Review.. 